Original paper

\title{
In-vivo EPID dosimetry for IMRT and VMAT based on through-air predicted portal dose algorithm
}

\author{
M.A. Najem ${ }^{\mathrm{a}, *}$, M. Tedder ${ }^{\mathrm{b}}$, D. King ${ }^{\mathrm{a}}$, D. Bernstein ${ }^{\mathrm{a}}$, R. Trouncer ${ }^{\mathrm{a}}$, C. Meehan ${ }^{\mathrm{a}}$, A.M. Bidmead ${ }^{\mathrm{a}}$ \\ a Joint Department of Physics, The Royal Marsden NHS Foundation Trust and The Institute of Cancer Research, Fulham Road, London SW3 6JJ, UK \\ ' Medical Physics Department, Guy's and St Thomas' NHS Foundation Trust, London SE1 7EH, UK
}

\section{A B S T R A C T}

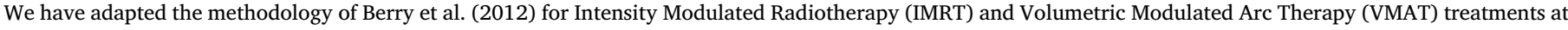

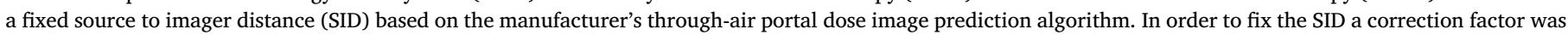

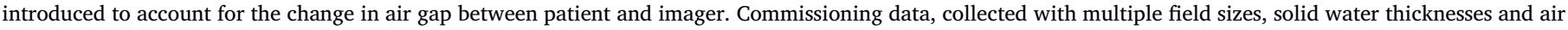

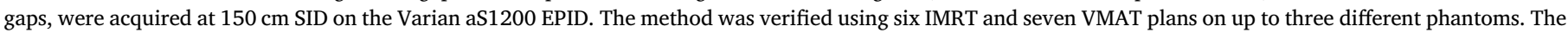

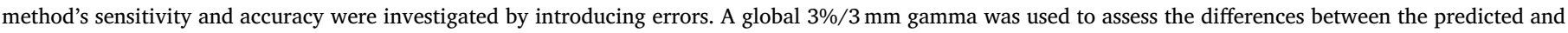

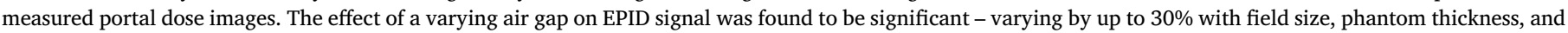

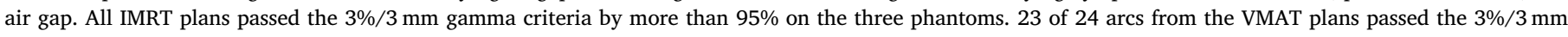

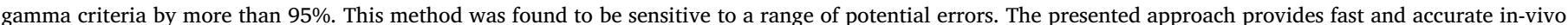
EPID dosimetry for IMRT and VMAT treatments and can potentially replace many pre-treatment verifications.

\section{Introduction}

Intensity Modulated Radiotherapy (IMRT) and Volumetric Modulated Arc Therapy (VMAT) enable the delivery of highly conformal and uniform dose distributions to planning target volume (PTV) whilst sparing organs at risk (OARs) [1]. While this enhanced delivery capability has been shown to be advantageous with respect to improving patient outcome $[2,3]$, it does introduce new challenges for the quality assurance (QA) of treatments in term of additional linac QA as well as patient-specific QA, which is recommended to be carried out prior to, or within the first few fractions of treatment [4,5]. In vivo dosimetry is a form of patient-specific QA that offers certain advantages over pre-treatment QA, namely that it actually verifies what is really of interest: the dose delivered to the patient. Within 4 years at the Netherland Institute of Cancer Research, more than 4000 plans were verified using an in-vivo dosimetry system. Of the 17 serious errors detected, 9 would not have been detected with pre-treatment verification [6]. The same conclusion was obtained from another study by the same institute carried out on more than 15,000 plans. 35 serious errors were detected that would not have been detected with pre-treatment verification due to mainly changes in patients anatomy [7].

EPID dosimetry can offer advantages in terms of the ease and speed with which in vivo dosimetry can be performed, the extra information afforded by sampling the entire radiation field rather than just a single point (as by using thermo-luminescent detectors (TLDs) or diodes), and the additional scope it offers for detecting changes in patient anatomy [8-10]. EPID dosimetry can be performed in many different ways, an overview of which is given in the literature review by Van Elmpt et al. [11]. Within the subcategory of in vivo EPID dosimetry several implementations have been developed each of which verifies the dose to the patient in different ways. Several in-vivo EPID software solutions have been commercially available recently and have been assessed in a number of studies $[12,13]$. However, the solutions thus far have mainly been developed in house by academic centres, resulting in a wide variety of methodologies. These methodologies can be divided into: a point dose verification in the patient, $2 \mathrm{D}$ transit dose verification at the level of the EPID, 2D transit dose verification in a plane in the patient or 3D transit dose verification in the patient [14-19]. A recent study by Bedford et al. (2017) investigated the agreement between the forward and back-projection transit EPID dosimetry for prostate radiotherapy. They found a fairly similar response from both methods and they concluded that both of them can be used to verify the dose delivered to the patient [20].

Van Elmpt et al. (2005) developed a system that predicted a transit portal dose image using a through air portal dose image and the radiological thickness of the path of the beam through the patient [21].

Berry et al. (2012) used a similar technique, but rather than using a measured through air portal dose image, they used the image predicted

\footnotetext{
* Corresponding author.

E-mail address: maan.najem@rmh.nhs.uk (M.A. Najem).
} 
by the Varian Portal Dosimetry software, which is based on the work of Van Esch et al. [15,22]. However, in their model, a fixed air gap of $35 \mathrm{~cm}$ was used between the phantom exit and EPID. The fixed air gap causes several problems. Firstly, it increases the treatment time for each patient as the EPID needs to be moved for each beam in order to keep a constant air gap which has several consequences including increased risk of intra-fraction motions and reduced patient throughput. Secondly, it increases the generation time of the portal dose images through air since each beam requires a verification plan with a different source to imager distance (SID) to be created in the Eclipse treatment planning system (TPS). Finally, it is not suitable for VMAT treatments as EPID needs to be at a fixed SID while the gantry rotates. Another limitation of the original methodology used by Berry et al. was that they did not include the couch model in their calculations, and consequently reported a decrease in the mean gamma pass rate for fields that transit the couch [23].

The aim of this work is to extend the methodology of Berry et al. (2012) to be used for both IMRT with a fixed SID and VMAT treatments. This is achieved by introducing a correction factor which takes into account the change in air gap between the patient exit and EPID for a fixed SID. To improve the accuracy of the system, the couch model was included in our calculation to take into account the couch attenuation at any gantry angle. In addition, a new approach to calculate IMRT and VMAT equivalent square field size is presented.

\section{Materials and methods}

\subsection{Equipment}

Portal dose images were delivered on a Varian TrueBeam linac which is equipped with the Varian portal imager aS1200. This imager has a sensitive area covering a $40 \times 40 \mathrm{~cm}^{2}$ field size at a $100 \mathrm{~cm}$ SID and high resolution $(1190 \times 1190$ pixels $)$ in the dosimetry mode. In addition, it has a backscatter shield to remove the effect of uneven backscatter from the support arm [24]. Portal dose images were acquired at dose rate up to $600 \mathrm{MU} / \mathrm{min}$ (the max dose rate for $6 \mathrm{MV}$ beam) on this machine.

The imager was calibrated according to the manufacturer's recommendation and the central pixel value calibrated to equal $0.444 \mathrm{CU}$ (calibrated unit) when irradiated with $100 \mathrm{MU}$ and a $10 \times 10 \mathrm{~cm}^{2}$ field at $150 \mathrm{~cm}$ SID. All measured portal dose images ( $\mathrm{mIs}$ ) in our experiments were acquired at a $150 \mathrm{~cm}$ SID. Three phantoms were used in the verification: A $30 \times 30 \times 19 \mathrm{~cm}^{3}$ water-equivalent "solid water (SW)" phantom, the RT01 phantom and the BrainLab pelvis phantom (BrainLab Medical Systems, Westchester, IL) (see Fig. 1) [25]. In addition, slabs of tissue-equivalent materials (water, bone and lung) were used to create phantoms with a range of complexities in order to test the accuracy of the presented model.

The predicted portal dose images through air $\left(p I s_{\text {air }}\right)$ were created using Eclipse v13.7. For an in-depth description of how $p I s_{\text {air }}$ are calculated, see the paper by Van Esch et al. [22]. The RT plan, RT
Table 1

A brief summary of each term in Eq. (1).

\begin{tabular}{ll} 
Parameter & Description \\
\hline$T$ & $\begin{array}{l}\text { Transmission factor which takes into account the attenuation of the } \\
\text { beam through the phantom }\end{array}$ \\
& $\begin{array}{l}\text { The off-axis pixel response function, that accounts for the fact that } \\
\text { the off axis spectrum of the incident beam varies as a function of } \\
\text { radial distance from the central axis }\end{array}$ \\
& $\begin{array}{l}\text { The air gap factor which corrects the EPID response due to change in } \\
\text { the distance between the patient exit and EPID }\end{array}$
\end{tabular}

structure, CT images and $p I s_{\text {air }}$ are imported to in-house software written in Python v3.5.1 in order to calculate the predicted images through patient $\left(p I s_{p}\right)$.

\subsection{Predicted images through patient model}

The $p I_{p}$ for any field size (FS), thickness $(t)$, air gap $(g)$ and fixed SID can be calculated from the $p s_{\text {air }}$ at any position (x, y) on the EPID using the following equation:

$p I_{p}=p I_{\text {air }} \cdot T(x, y, F S, t) \cdot O A R(x, y, t) \cdot G(x, y, F S, t, g)$

Each of the terms in Eq. (1) is briefly described in Table 1 below.

\subsubsection{Transmission correction factor model}

In the implementation used by Berry et al. (2012), two terms were used to account for the two primary causes of attenuation of the beam through the phantom: the attenuation of the primary beam, which was modelled in narrow beam conditions using Monte Carlo (MC) simulations, and the effect of scattered and secondary radiation, which was empirically derived by measuring the EPID response for several field sizes and SW block thicknesses [15]. In this work, both terms are combined into one empirically derived correction factor dependent on attenuator thickness and field size. A series of images were acquired with the EPID for different field sizes and thicknesses of SW blocks to determine the equation that defines this correction factor. The data were fitted using a nonlinear least squares method to the following equation:

$T(t, F S)=\frac{A(F S) e^{-B(F S) t}+C(F S) e^{-D(F S) t}}{S(0, F S)}$

where $A(F S)=a_{1} e^{-a_{2} F S}+a_{3}, B(F S)=b_{1} F S^{b_{2}}, C(F S)=c_{1} e^{-c_{2} F S}+c_{3}$, $D(F S)=d_{1} \mathrm{FS}^{\mathrm{d}_{2}}$.

$S(0, F S)$ is the mean signal in the $4 \times 4$ pixels at the EPID centre produced by the beam with no attenuation for a given field size. $a_{1}, a_{2}, a_{3}, b_{1}, b_{2}, c_{1}, c_{2}, c_{3}, d_{1}, d_{2}$ are empirically determined fitting parameters. Table 2 summarises the commissioning measurements performed to determine the $\mathrm{T}$ factor. The model was then verified with a mix of field sizes and SW thicknesses.

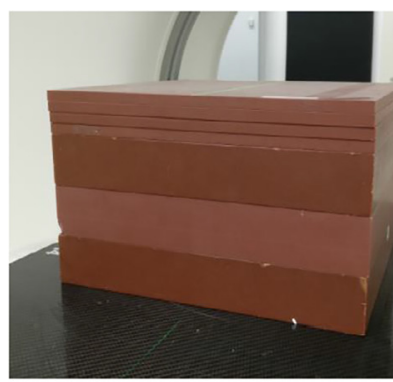

(a) $19 \mathrm{~cm}$ solid water phantom

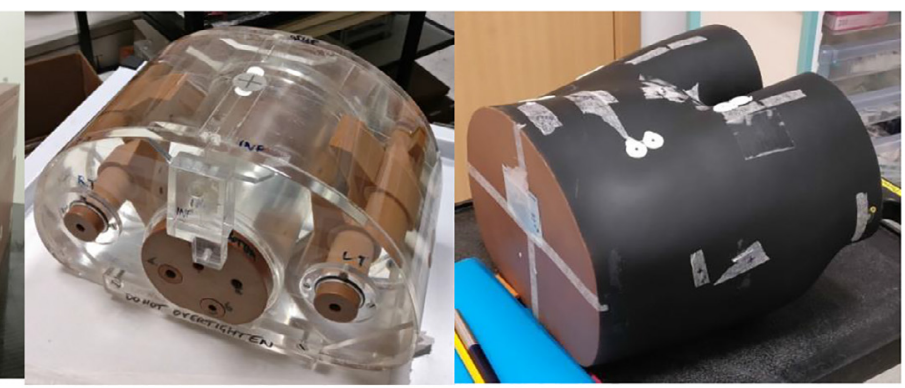

(b) RT01 phantom (c) BrainLab pelvis phantom

Fig. 1. Phantoms used to verify our method: (a) a $19 \mathrm{~cm}$ solid water phantom, (b) the RT01 phantom and (c) the BrainLab pelvis phantom. 
Table 2

The commissioning measurements used to model the $\mathrm{T}$ factor.

\begin{tabular}{lllll}
\hline Field size $\left(\mathrm{cm}^{2}\right)$ & $\begin{array}{l}\text { Solid water } \\
\text { thickness }(\mathrm{cm})\end{array}$ & $\begin{array}{l}\text { Air gap } \\
(\mathrm{cm})\end{array}$ & $\begin{array}{l}\text { Gantry } \\
(\mathrm{deg})\end{array}$ & MUs \\
\hline $3 \times 3,5 \times 5,8 \times 8$, & $0,5,10,15,20$, & 40 & 270 & 100 \\
$10 \times 10,15 \times 15$, & $25,30,35,40$ & & & \\
$20 \times 20,25 \times 25$ & & & & \\
\hline
\end{tabular}

\subsubsection{Off-axis pixel response factor model}

This correction factor is derived empirically, using a similar set of data as for the $\mathrm{T}$ correction factor, but with only a field size that fully covers the EPID at $150 \mathrm{~cm}$ SID $\left(27 \times 27 \mathrm{~cm}^{2}\right)$. A third-degree polynomial is used to fit the OAR factor for each individual pixel as a function of the attenuator thickness as described in the following equation:

$O A R(i, j)=\alpha_{1}(i, j) t(i, j)^{3}+\alpha_{2}(i, j) t(i, j)^{2}+\alpha_{3}(i, j) t(i, j)+\alpha_{4}(i, j)$

For a given pixel at the $\mathrm{i}^{\text {th }}$ row and $\mathrm{j}^{\text {th }}$ column $\alpha_{1}(i, j), \alpha_{2}(i, j), \alpha_{3}(i, j), \alpha_{4}(i, j)$ are empirically determined fitting parameters and $t(i, j)$ is the equivalent radiological path length traced through the CT scan to the pixel.

Table 3 summarises the commissioning measurements performed to determine the OAR factor.

\subsubsection{Air gap correction factor model}

A series of portal dose images were acquired with the EPID for different field sizes, thicknesses of SW blocks and air gaps to determine the correct model for this factor. In this work, the $\mathrm{G}$ factor is divided into two terms:

$G(x, y, t, F S, g)=G_{c}(t, F S, g) G_{o}(x, y, g)$

where $G_{c}(t, F S, g)$ is the air gap factor at the centre region (the $4 \times 4$ pixels at EPID centre) of the EPID which is a function of thickness, field size and air gap. $G_{0}(x, y, g)$ is the air gap off-axis pixel response function which takes into account the change in off-axis pixel response due to the change in air gap distance. Both factors were normalised to a $40 \mathrm{~cm}$ air gap. A multi-dimensional look-up table to interpolate between field sizes, thicknesses, and air gaps is used to model the $G_{c}$ factor. The $G_{o}$ factor was fitted using a nonlinear least squares method to the following equation:

$G_{o}(x, y, g)=e^{-\Delta(g) \cdot \Omega(x, y)}$

where: $\Delta(g)=\beta_{1}\left(g-g_{0}\right)^{2}+\beta_{2}\left(g-g_{0}\right)+\beta_{3}, \Omega(x, y)=\frac{\gamma_{1}\left(x^{2}+y^{2}\right)}{\gamma_{2}}$.

where $\beta_{1}, \beta_{2}, \beta_{3}, \gamma_{1}, \gamma_{2}$ are the empirically determined fitting parameters and $g_{0}$ is the default air gap at which the other correction factors were modelled. In this work, $g_{0}=40 \mathrm{~cm}$. Table 4 summarises the commissioning measurements used to determine the $\mathrm{G}$ factor.

\subsubsection{The equivalent thickness map}

In order to predict the effect of patient (phantom) attenuation on $p I_{\text {air }}$, information about the radiological path length for each photon beam arriving at the EPID is required. The patient CT scan is used along with a calibration table which converts from Hounsfield Units (HU) to electron density to calculate electron density at each voxel in the CT scan. The body outline from the structure set is used to restrict the

Table 3

The commissioning measurements used to model the OAR factor.

\begin{tabular}{lllll}
\hline Field size $\left(\mathrm{cm}^{2}\right)$ & Slabs thickness $(\mathrm{cm})$ & $\begin{array}{l}\text { Air Gap } \\
(\mathrm{cm})\end{array}$ & Gantry $(\mathrm{deg})$ & Mus \\
\hline $27 \times 27$ & $\begin{array}{l}0,1,2,3,5,10,15,20,25, \\
30,35,40\end{array}$ & 40 & 0 & 100 \\
& & &
\end{tabular}

Table 4

The acquisition parameters used to model the $\mathrm{G}$ factor.

\begin{tabular}{cllll} 
Field size $\left(\mathrm{cm}^{2}\right)$ & $\begin{array}{l}\text { Slabs thickness } \\
(\mathrm{cm})\end{array}$ & Air gap $(\mathrm{cm})$ & $\begin{array}{l}\text { Gantry } \\
(\mathrm{deg})\end{array}$ & MUs \\
\hline $5 \times 5,10 \times 10$, & $\begin{array}{l}0,10,15,20,25, \\
15 \times 15,20 \times 20\end{array}$ & $\begin{array}{l}20,25,30,35, \\
40,35,40\end{array}$ & $\begin{array}{l}40,(45,50, \\
55)^{*}\end{array}$ & 100 \\
& & & \\
\hline
\end{tabular}

* If possible due to machine interlock for large air gaps and solid water blocks.

calculated voxels to just those within the body. The couch structure, generated in the Eclipse TPS, is also included in the calculation so that the attenuation through the couch at any gantry angle is included. The radiological path length is then calculated for each ray arriving at a pixel at the EPID using the method reported by Siddon [26]. The ray tracing algorithm was verified using virtual phantoms with known geometries and densities as well as comparing the equivalent thickness at the central axis for each verification plan to that obtained from Eclipse TPS. The calculated equivalent thicknesses were found to agree with those calculated in Eclipse to within $\pm 1 \mathrm{~mm}$.

\subsubsection{Equivalent square field size for dynamic fields}

To assess the impact of both the collimator and MLC positions on the estimation of the equivalent square field size, we investigated if the equivalent square field size for a given dynamic field can be calculated from only the field formed by X and Y secondary collimators. Several dynamic fields with the same $\mathrm{X}$ and $\mathrm{Y}$ secondary collimator (i.e. $10 \times 10 \mathrm{~cm}^{2}$ ) opening but different fluence map were delivered through a $20 \mathrm{~cm}$ thick SW phantom at three different air gaps: 20, 30 and $40 \mathrm{~cm}$. Fig. 2 shows the fluence map for each dynamic field. It was found that the correction factors depend on both total fluence and open area and not only on the $\mathrm{X}$ and $\mathrm{Y}$ collimator size. The equivalent square field size $F S$, which gives the best agreement with our measurements, was found to be calculated using the following empirical equation:

$F S=F S_{O F} \sqrt{\frac{A_{f}}{A_{O F}}}$

where $F S_{O F}$ is the equivalent square field size calculated from $\mathrm{X}$ and $\mathrm{Y}$ collimators, $A_{f}$ is the area in the field where the fluence is larger than zero, and $A_{O F}$ is the area within the field formed by $\mathrm{X}$ and $\mathrm{Y}$ collimators.

\subsection{Predicted images through patient model verification}

Six IMRT plans and seven VMAT plans were used to verify the presented method. The IMRT plans were clinical prostate and seminal vesicle plans; two were planned according to the CHHiP trial protocol, two to the PACE protocol, and two according to the pre-CHHiP clinical standard at RMH $[27,28]$. The VMAT plans consisted of prostate bed $(\mathrm{n}=2)$, prostate and nodes $(\mathrm{n}=2)$, larynx bed $(\mathrm{n}=2)$, and vagina $(\mathrm{n}=1)$ plans. Each plan was delivered on up to three different phantoms described in Section 2.1. In total, 15 IMRT fields were delivered to the RT01 phantom, 15 IMRT fields and 12 VMAT arcs were delivered to the $19 \mathrm{~cm}$ solid water phantom, and 30 IMRT fields and 12 VMAT arcs were delivered to the BrainLab pelvis phantom. $p I s_{p}$ and $m I s$ were compared using a global $3 \% / 3 \mathrm{~mm}$ gamma evaluation with $10 \%$ dose threshold as it is the most common gamma criteria used in dosimetric QA [29].

In addition, $p I s_{p}$ and $m I s$ were compared using a local $2 \% / 2 \mathrm{~mm}$ Gamma criteria to test the sensitivity of the system [29].

All plans used were independently verified using PTW OCTAVIUS 1500 ionisation chamber array (PTW, Freiburg, Germany) and/or Varian pre-treatment verification (Portal Dosimetry) software. All plans passed the local $3 \% / 3 \mathrm{~mm}$ gamma evaluation by more than $98 \%$ on the PTW array and by more than $99 \%$ on Varian pre-treatment verification 

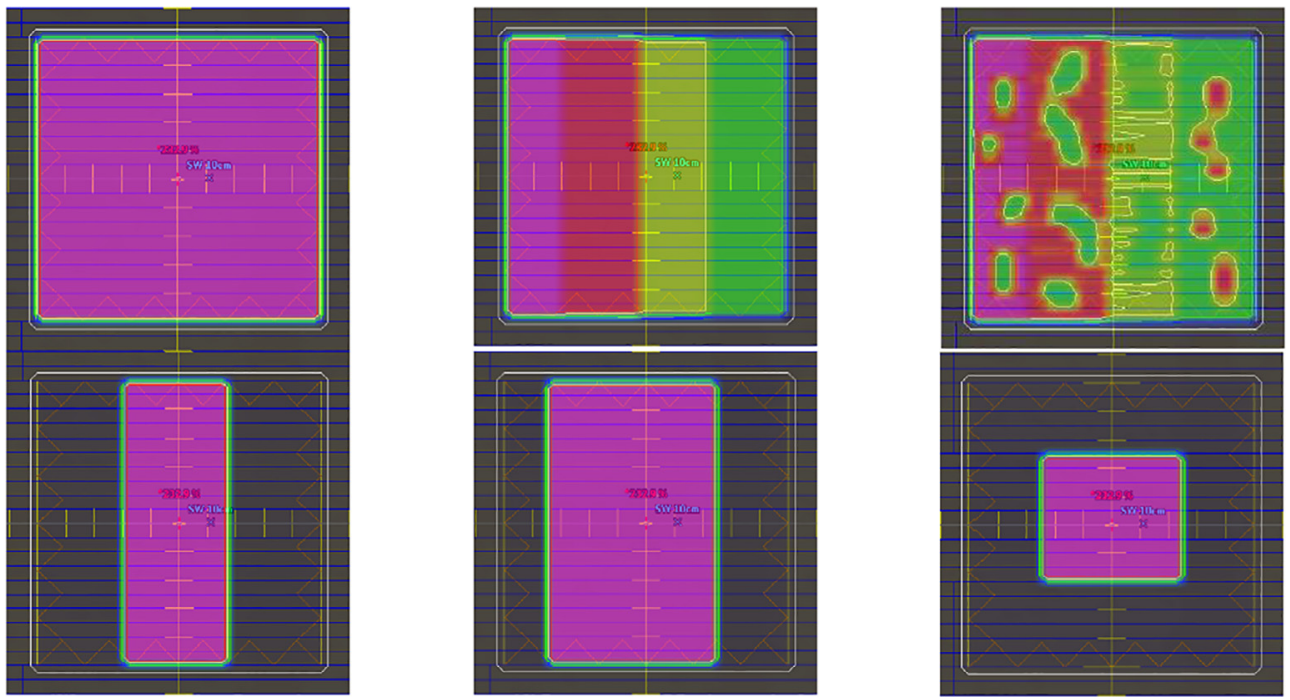

Fig. 2. The fluence maps used to determine the equivalent square field size for a dynamic field.

software.

\subsubsection{The effect of heterogeneity}

To investigate the ability of our model to predict accurate portal dose images in the presence of heterogeneities, an anterior IMRT field from one of the six prostate plans was delivered on several phantoms (shown in Fig. 3). These phantoms were made of tissue-equivalent materials; water (WT1, relative electron density $=1.0$ ), cortical bone $(\mathrm{SB} 5$, relative electron density $=1.73$ ) and lung (LN10, relative electron density $=0.3$ ) [30] with a range of complexities similar to what was presented by the work of Berry et al. [15].

The following geometries and material configurations were

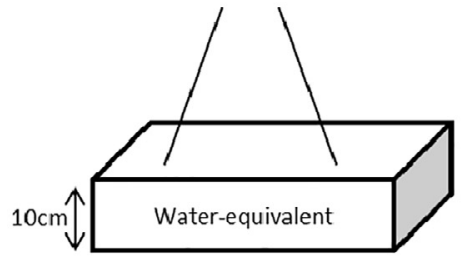

(a)

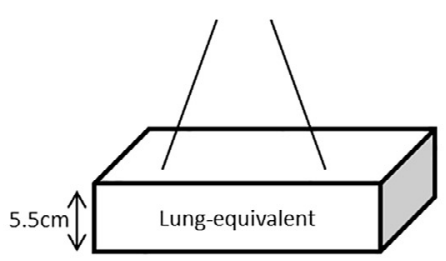

(d)

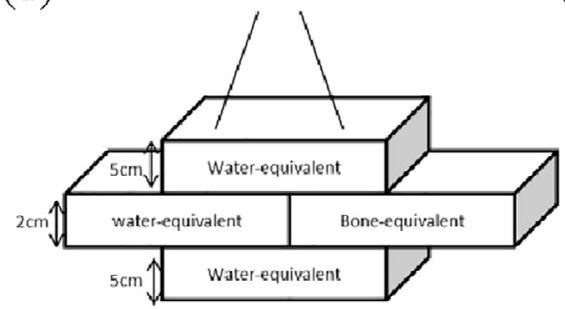

$(\mathrm{g})$

Fig. 3. Diagrams of the slab phantoms used to test our model using a range of homogeneous and inhomogeneous geometries, with materials of varying electron densities. a, c, d: A field pointing directly at a $10 \mathrm{~cm}$ thick slab of WT1, $5 \mathrm{~cm}$ thick slab of SB5, $5.5 \mathrm{~cm}$ thick slab of LN10, respectively. b: A field pointing directly and beam passing through part of a $10 \mathrm{~cm}$ thick slab of WT1. e, f: A field pointing directly and passing through multiple materials (WT1-LN10-WT1 and WT1-SB5-WT1, respectively) with tissue boundaries along the ray path. $\mathrm{g}$, h, i: A field pointing directly and passing through multiple materials (WT1-[WT1-SB5]-WT1, WT1-[WT1LN10]-WT1 and WT1-[SB5-LN10]-WT1, respectively) with tissue boundaries along the ray path and perpendicular to the treatment field. (c)

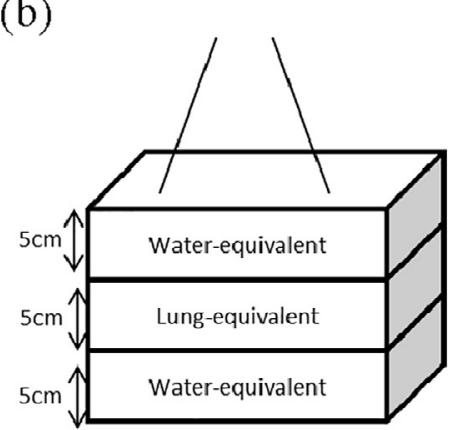

(e)

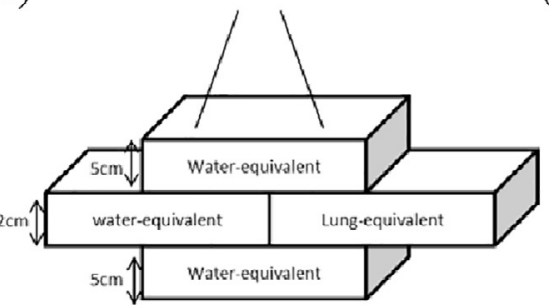

(f)

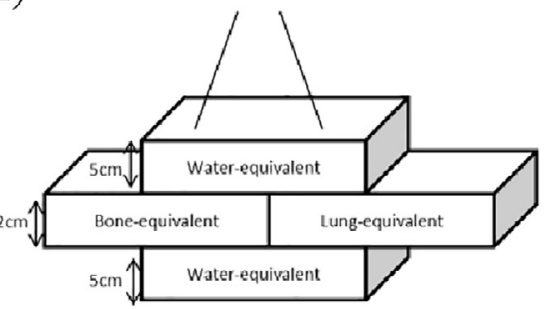

(i) 
measured with the field pointing directly at the phantom: single materials (Fig. 3a-d), single material with a beam passing through part of the phantom only (Fig. 3b), multiple materials with tissue boundaries along the ray path (Fig. 3b-f), multiple materials with tissue boundaries both along the ray path and perpendicular to the treatment field (Fig. 3g-i)

\subsection{Model sensitivity}

\subsubsection{Error detection}

In order to test the sensitivity of our model to detect dose delivery errors in the patient, several types of errors were deliberately introduced either into the plan itself or the phantom setup. This was done using a single IMRT verification plan and followed an approach similar to that of Bedford et al. [14]. The $p I s_{p}$ for the unaltered plan and setup were compared to the $m I s$ for the delivery containing the errors, to test whether the dose discrepancy is greater than that observed for the unaltered IMRT plan and therefore determine whether the error can actually be detected.

The deliberate errors were all introduced to a prostate IMRT plan, created in accordance with the CHHiP trial protocol. The error plans were all delivered to the BrainLab pelvis phantom apart from in the case of the change in phantom size, which was done using SW blocks. The errors introduced were:

1. Dose errors: The number of monitor units for all fields in the plan was altered by $-5 \%,+1 \%,+3 \%,+5 \%$ and $+10 \%$, creating five new plans with deliberate errors in. All other aspects of the plan were kept the same.

2. Gantry angle error: Two plans were created, identical to the original plan on the BrainLab pelvis phantom, except with gantry angle offsets of $+5^{\circ}$ and $+10^{\circ}$ introduced.

3. Patient set-up errors: One field from the original plan (the left anterior oblique field, at a gantry angle of $35^{\circ}$ ) was delivered to the BrainLab pelvis phantom, but with the phantom offset by $0.5 \mathrm{~cm}$, $1 \mathrm{~cm}, 1.5 \mathrm{~cm}$ and $2 \mathrm{~cm}$ laterally towards the patient's right, and by the same offsets in the anterior direction, resulting in 8 deliveries of one field.

4. Change in phantom size: For this sensitivity test, the posterior field $\left(180^{\circ}\right)$ from the original plan was delivered to the CT scanned $19 \mathrm{~cm}$ solid water block, with $0.5 \mathrm{~cm}, 1 \mathrm{~cm}, 2 \mathrm{~cm}$ and $3 \mathrm{~cm}$ of solid water added on top; only the posterior field was measured to save time, as this was the field that would be most affected by a change in depth of the phantom.

5. MLC errors: new versions of the original plan were created with MLC errors introduced by altering the MLC plan file using a python script. The MLC files were then imported back into Eclipse, and the plan was calculated again using the new MLC positions. The type of MLC errors and the fields which they were applied to is detailed in

Table 5

The MLC errors that were deliberately introduced into each field of one of IMRT plans in order to test the sensitivity of portal dosimetry to different types of MLC error.

\begin{tabular}{|c|c|c|}
\hline Error & $\begin{array}{l}\text { Field (Gantry angle } \\
{[\mathrm{deg}] \text { ) }}\end{array}$ & MLC error \\
\hline E1 & All fields & Both banks shifted in the same direction by $5 \mathrm{~mm}$ \\
\hline E2 & $\operatorname{POST}\left(180^{\circ}\right)$ & Shift both leaf banks in by $2 \mathrm{~mm}$ \\
\hline E3 & $\operatorname{LPO}\left(100^{\circ}\right)$ & $\begin{array}{l}\text { Shift the leaves of bank B that are within the field } \\
\text { in by } 5 \mathrm{~mm}\end{array}$ \\
\hline E4 & $\mathrm{LAO}\left(35^{\circ}\right)$ & $\begin{array}{l}\text { Shift one leaf in bank A that is close to the centre } \\
\text { of the field in by } 1 \mathrm{~cm}\end{array}$ \\
\hline E5 & RAO $\left(325^{\circ}\right)$ & $\begin{array}{l}\text { Open all leaf pairs that are within the field out by } \\
5 \mathrm{~mm}\end{array}$ \\
\hline E6 & $\mathrm{RPO}\left(260^{\circ}\right)$ & $\begin{array}{l}\text { Shift four leaves from bank } \mathrm{A} \text { at the superior edge } \\
\text { of the treatment field by } 1 \mathrm{~cm}\end{array}$ \\
\hline
\end{tabular}

Table 6

$\mathrm{T}$ factor fitting parameters.

$\begin{array}{lllll}a_{1}=-0.9580 & a_{2}=0.0185 & a_{3}=0.9529 & b_{1}=0.4143 & b_{2}=-0.7029 \\ c_{1}=-50.2717 & c_{2}=-0.0002 & c_{3}=50.6647 & d_{1}=0.0548 & d_{2}=-0.1675\end{array}$

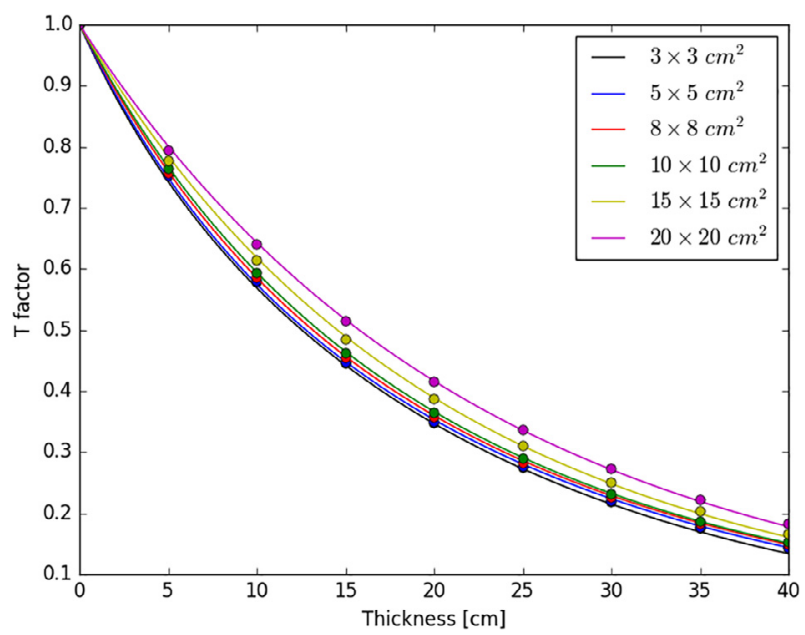

Fig. 4. The measured (circles) and modelled (lines) $\mathrm{T}$ factors as a function of solid water thicknesses for different field sizes.

Table 5.

\section{Results}

\subsection{Transmission correction factor}

The T factor was fitted successfully using Eq. (2). The $\mathrm{R}^{2}$ value of the fitted equation was found to be 0.9998 . The fitting parameters are listed in Table 6. Fig. 4 shows the measured and modelled $\mathrm{T}$ factor as a function of SW thickness for several field sizes. A comparison of the measured and modelled $\mathrm{T}$ factors for several field sizes and SW thicknesses is shown on Table 7 .

\subsection{Off-axis pixel response factor}

All measured and modelled OAR model images passed the $0.5 \%$ / $0.5 \mathrm{~mm} 2 \mathrm{D}$ gamma by $100 \%$. Fig. 5 shows the measured and modelled OAR factors as a function of SW thickness at different positions on the EPID.

\subsection{Air gap correction factor}

Fig. 6a shows the measured and modelled $G_{c}$ factors for a $15 \times 15 \mathrm{~cm}^{2}$ field size as a function of SW thickness at different air gaps. Fig. $6 \mathrm{~b}$ shows $G_{c}$ factor for $30 \mathrm{~cm}$ solid water thickness as a function of field size for different air gaps.

A comparison of the measured and modelled $G_{c}$ factors for several field sizes, SW thicknesses and air gaps is shown in Table 8.

Table 7

The modelled and measured $\mathrm{T}$ factors for different field sizes and SW thicknesses.

\begin{tabular}{lllll}
\hline Field Size $(\mathrm{cm})$ & Thickness $(\mathrm{cm})$ & Measured (CU) & Modelled (CU) & Diff (\%) \\
\hline $25 \times 25$ & 2.84 & 0.419 & 0.4191 & 0.0 \\
$7 \times 18$ & 17 & 0.187 & 0.1865 & -0.3 \\
$13 \times 13$ & 17 & 0.197 & 0.1968 & -0.1 \\
$7 \times 14$ & 23.84 & 0.134 & 0.1337 & -0.2 \\
$15 \times 15$ & 0.84 & 0.438 & 0.4385 & 0.1 \\
\hline
\end{tabular}




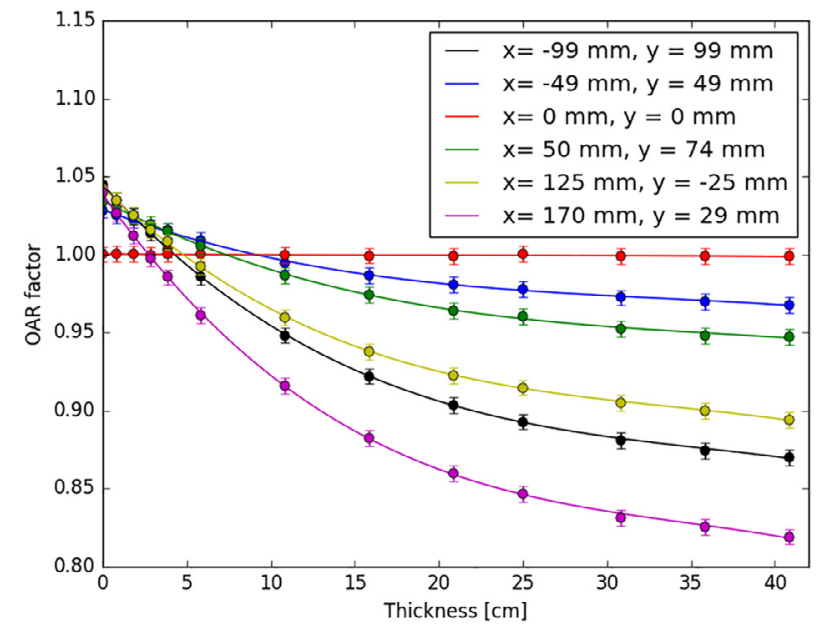

Fig. 5. The measured (circle) and modelled (line) OAR factors as a function of solid water thicknesses at different locations on the EPID. Note the error bars illustrate a $0.5 \%$ difference in OAR value at each point.

Fig. $7 \mathrm{a}$ and b show the $G_{0}$ factor along the x-axis at $30 \mathrm{~cm}$ air gap for a $15 \times 15 \mathrm{~cm}^{2}$ field size and different SW thicknesses and $20 \mathrm{~cm}$ solid water thickness and different field sizes, respectively. Fig. 7c shows the measured and modelled $G_{0}$ factor along the x-axis as a function of air gap for a $20 \times 20 \mathrm{~cm}^{2}$ field size and $20 \mathrm{~cm}$ SW thickness. Fig. $7 \mathrm{~d}$ shows the difference between the modelled and measured $G_{o}$ factor for different air gaps. Table 9 lists the fitting parameters used to fit Eq. (5). The $\mathrm{R}^{2}$ value of the fitted equation was found to be 0.9994 .

\subsection{Verification results}

\subsubsection{IMRT plans}

All IMRT fields (60 fields in total) passed the $3 \% / 3 \mathrm{~mm}$ gamma criteria by more than $95 \%$. The average gamma pass rate was $99.8 \pm 0.3(1 \mathrm{SD}), 99.9 \pm 0.3(1 \mathrm{SD})$ and $99.3 \pm 1.2(1 \mathrm{SD})$ for $19 \mathrm{~cm}$ SW, RT01 and BrainLab pelvis phantoms, respectively. Fig. 8 shows a histogram of gamma pass rate for all IMRT fields on the three phantoms. The average gamma pass rate for beams which do and do not pass through the couch was found to be the same (99.6 $\pm 0.9(1 \mathrm{SD})$ ). 45 of 60 fields passed the local $2 \% / 2 \mathrm{~mm}$ gamma criteria by more than $95 \%$. The overall global $3 \% / 3 \mathrm{~mm}$ and local $2 \% / 2 \mathrm{~mm}$ gamma pass rate and mean gamma for all IMRT fields are listed in Table 10.

\subsubsection{VMAT plans}

For VMAT plans, 23 of the 24 arcs passed the $3 \% / 3 \mathrm{~mm}$ gamma

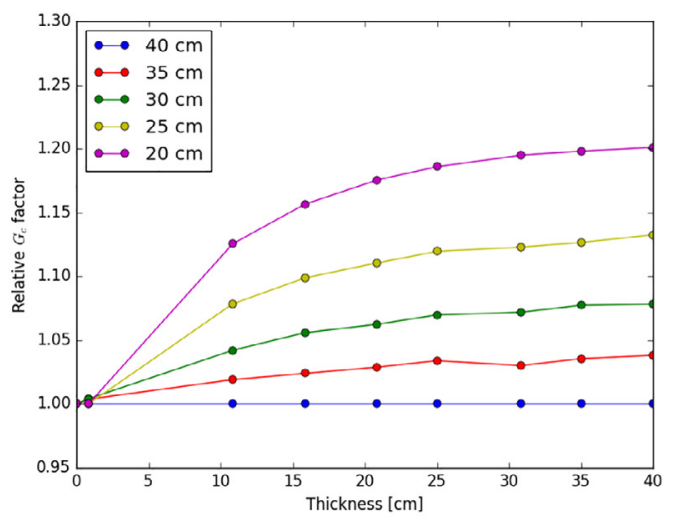

(a)
Table 8

The modelled and measured $\mathrm{G}_{\mathrm{c}}$ factors for different field sizes, SW thicknesses and air gaps.

\begin{tabular}{llllll}
\hline FS $\left(\mathrm{cm}^{2}\right)$ & $\mathrm{t}(\mathrm{cm})$ & $\mathrm{g}(\mathrm{cm})$ & Measured & Modelled & Diff (\%) \\
\hline 13 & 20.84 & 35 & 1.024 & 1.024 & 0.0 \\
13 & 20.84 & 30 & 1.054 & 1.052 & -0.2 \\
13 & 20.84 & 20 & 1.151 & 1.149 & -0.1 \\
13 & 20.84 & 37 & 1.012 & 1.014 & 0.2 \\
7.9 & 30.46 & 35 & 1.019 & 1.013 & -0.6 \\
7.9 & 30.46 & 30 & 1.029 & 1.032 & 0.4 \\
10 & 9.34 & 35 & 1.011 & 1.010 & -0.1 \\
10 & 9.34 & 30 & 1.022 & 1.022 & 0.0 \\
\hline
\end{tabular}

criteria by more than $95 \%$. However, the $3 \% / 3 \mathrm{~mm}$ gamma pass rate of the failed arc was $94.6 \%$. The mean $3 \% / 3 \mathrm{~mm}$ gamma pass rate was $98.7 \pm 1.8(1 \mathrm{SD})$ and $98.9 \pm 1.5(1 \mathrm{SD})$ for the $19 \mathrm{~cm} \mathrm{SW}$ and BrainLab pelvis phantoms, respectively. Fig. 9 shows a histogram of gamma pass rate for all VMAT fields on the $19 \mathrm{~cm}$ SW and BrainLab pelvis phantoms.

None of the VMAT plans passed the local $2 \% / 2 \mathrm{~mm}$ gamma criteria by more than $95 \%$. The overall global $3 \% / 3 \mathrm{~mm}$ and local $2 \% / 2 \mathrm{~mm}$ gamma pass rate and mean gamma for all VMAT arcs are listed in Table 10. Fig. 10 shows the $p I_{p}$ and $m I s$ images and global $3 \% / 3 \mathrm{~mm}$ and local $2 \% / 2 \mathrm{~mm}$ gamma maps for a VMAT field delivered on the BrainLab pelvis phantom.

\subsubsection{The effect of heterogeneity}

All beams, delivered on phantoms shown in Fig. 3, passed the 3\%/ $3 \mathrm{~mm}$ gamma criteria by more than $99.5 \%$ (see Fig. 1 in the Supplementary material).

\subsection{Model sensitivity}

\subsubsection{Error detection}

Table 11 shows the $3 \% / 3 \mathrm{~mm}$ gamma pass rate for the plans with errors deliberately introduced that were delivered on the BrainLab pelvis phantom.

Table 12 shows the $3 \% / 3 \mathrm{~mm}$ gamma pass rate and mean gamma, mean dose difference and the dose difference at the centre of the EPID between the measured and predicted images for errors plans delivered on a $19 \mathrm{~cm}$ SW phantom.

\section{Discussion}

As can be seen in Fig. 4, the modelled $\mathrm{T}$ factor was in a good agreement with measured values. The difference between the measured and modelled $\mathrm{T}$ factor for different field sizes and SW thicknesses was

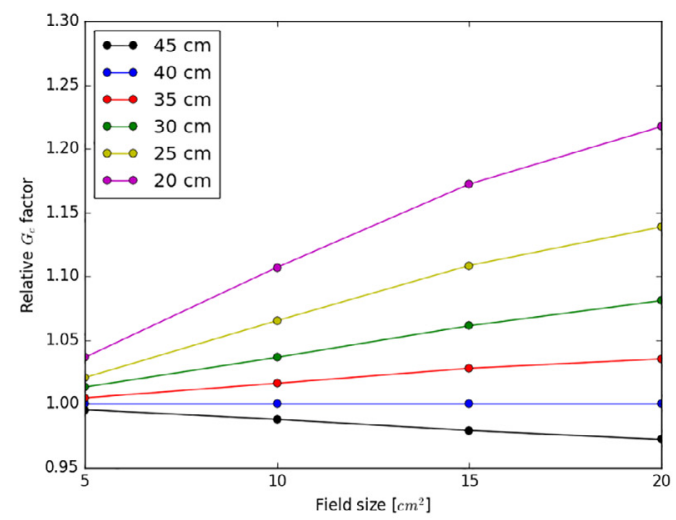

(b)

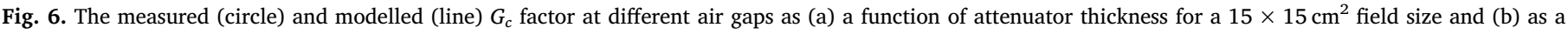
function of field size for a $30 \mathrm{~cm}$ solid water thickness. 


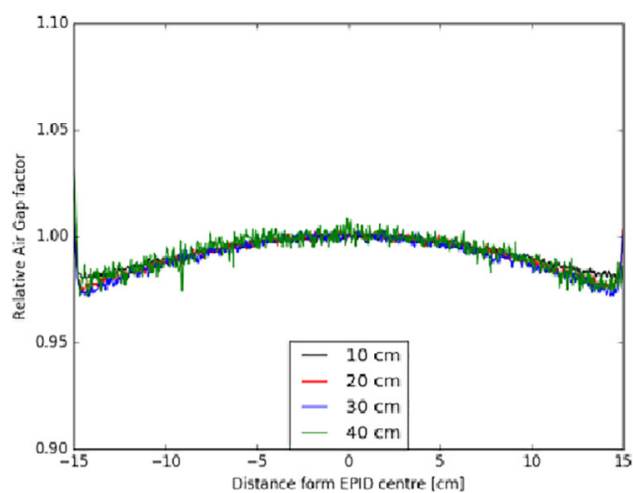

(a)

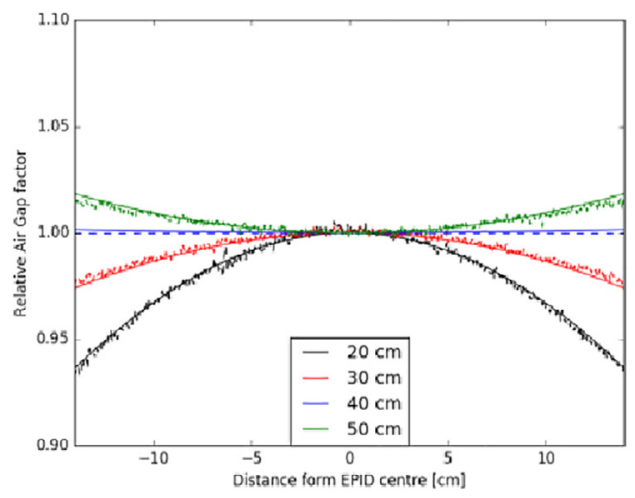

(c)

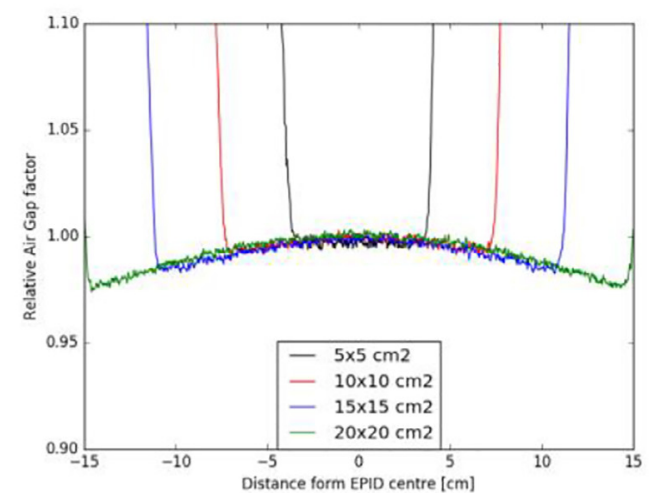

(b)

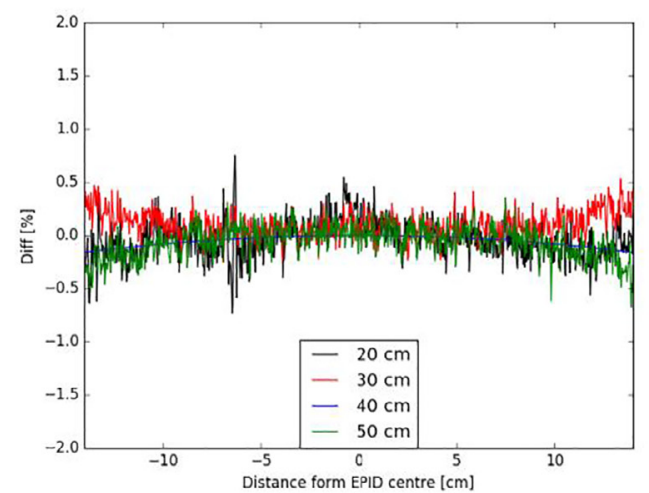

(d)

Fig. 7. The measured $G_{o}$ factor at a $30 \mathrm{~cm}$ air gap for (a) a $15 \times 15 \mathrm{~cm}^{2}$ field size and different thickness and (b) for a $20 \mathrm{~cm}$ solid water thickness and different field sizes. (c) The measured (dashed line) and modelled (solid line) $G_{o}$ factor along the x-axis for a $20 \times 20 \mathrm{~cm}^{2}$ field size, $20 \mathrm{~cm}$ SW thickness and several air gaps. (d) The percentage difference between the measured and modelled $G_{o}$ factor along the x-axis for different air gaps.

Table 9

The fitting parameters of $G_{o}$ factor.

\begin{tabular}{lllll}
\hline$\beta_{1}=7.590210^{-8}$ & $\beta_{2}=1.0211$ & $\beta_{3}=40.1247$ & $\gamma_{1}=-28.6866$ & $\gamma_{2}=26.7742$
\end{tabular}

found to be less than $0.5 \%$ as seen Table 7 . Berry et al. (2012) used two terms to model this factor and carried out several MC simulations to calculate the mass attenuation factor for a $6 \mathrm{MV}$ photon beam [15].
However, we found that using one term to model this factor was sufficient and easier to implement since no MC simulations are required.

With regards to OAR factor, the third-degree polynomial model at each individual pixel was found to accurately fit this factor (Fig. 5). All commissioning images passed by $100 \%$ the $0.5 \% / 0.5 \mathrm{~mm} 2 \mathrm{D}$ gamma evaluation. The third-degree degree polynomial model was found to give better agreement in our work compared with the Gaussian model introduced by Berry et al. (2012). This difference could be due to the

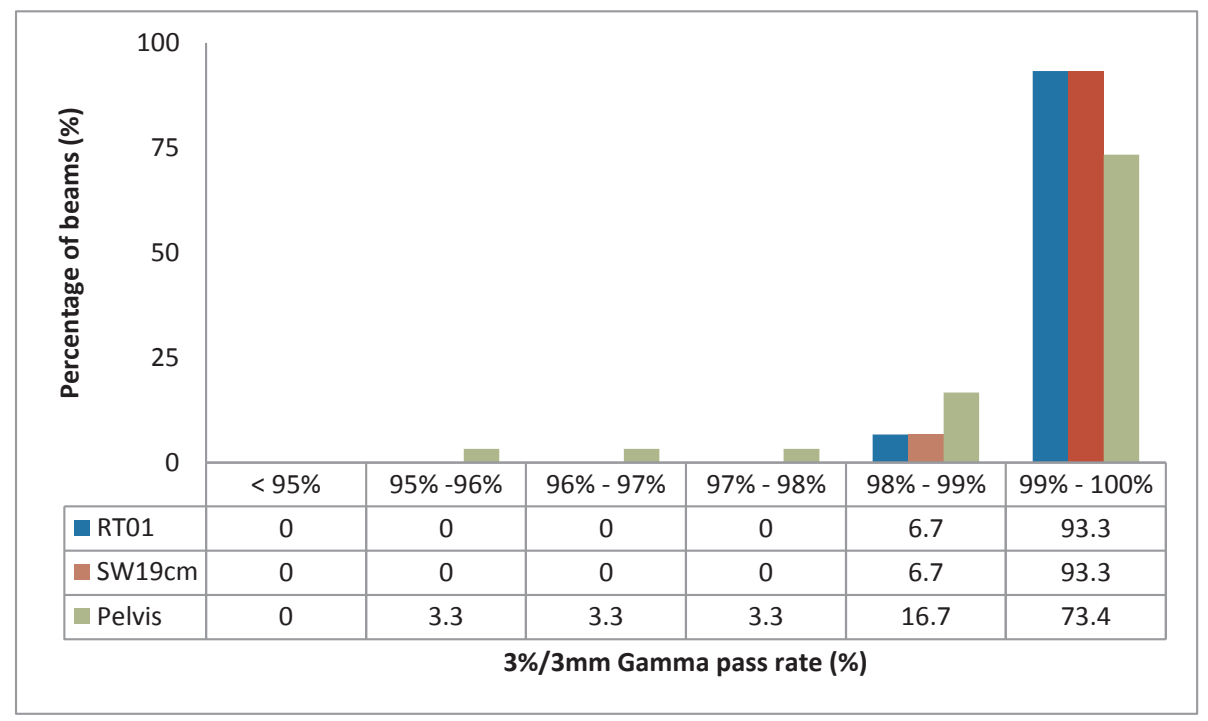

Fig. 8. Gamma pass rate histogram for IMRT fields delivered on the three phantoms. 
Table 10

The overall global $3 \% / 3 \mathrm{~mm}$ and local $2 \% / 2 \mathrm{~mm}$ gamma pass rate and mean gamma for all IMRT and VMAT plans.

\begin{tabular}{|c|c|c|c|c|}
\hline & \multicolumn{2}{|c|}{ Global 3\%/3 mm gamma } & \multicolumn{2}{|c|}{ Local $2 \% / 2 \mathrm{~mm}$ Gamma } \\
\hline & Pass rate $[\%]$ & Mean gamma & Pass rate $[\%]$ & Mean gamma \\
\hline IMRT & $99.6 \pm 0.9(1 S D)$ & $0.22 \pm 0.07(1 \mathrm{SD})$ & $96.1 \pm 5.5(1 \mathrm{SD})$ & $0.33 \pm 0.11(1 S D)$ \\
\hline VMAT & $98.8 \pm 1.7(1 \mathrm{SD})$ & $0.30 \pm 0.05(1 \mathrm{SD})$ & $84.5 \pm 6.7(1 \mathrm{SD})$ & $0.82 \pm 0.32(1 \mathrm{SD})$ \\
\hline
\end{tabular}

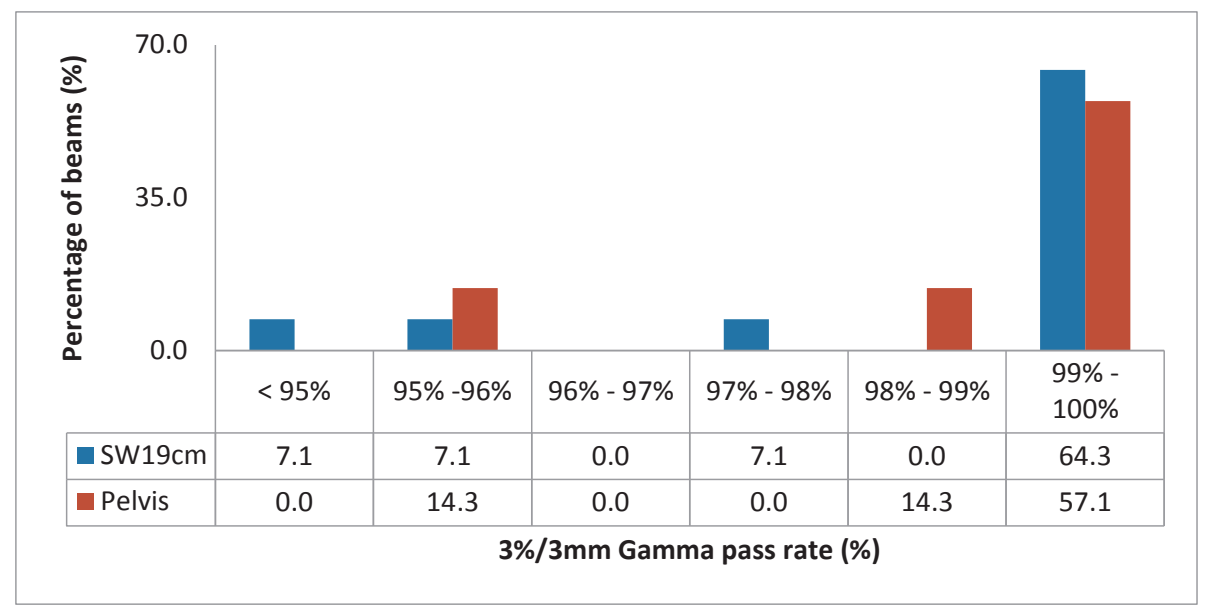

Fig. 9. $3 \% / 3 \mathrm{~mm}$ gamma pass rate histogram for VMAT fields delivered on the $19 \mathrm{~cm} \mathrm{SW}$ and BrainLab phantoms.

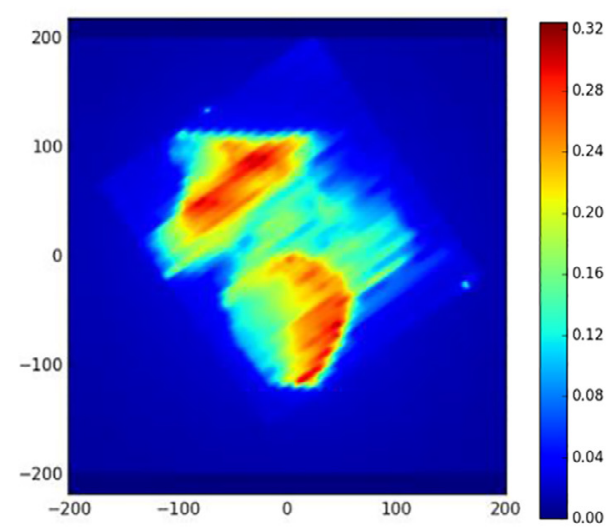

(a)

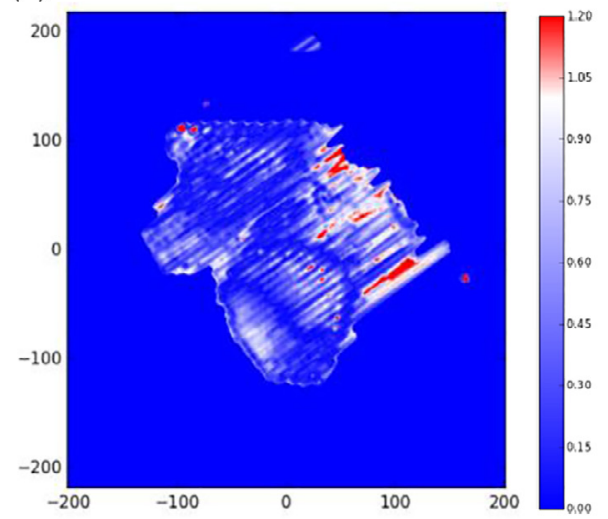

Pass rate $=95.8 \%$

(c)

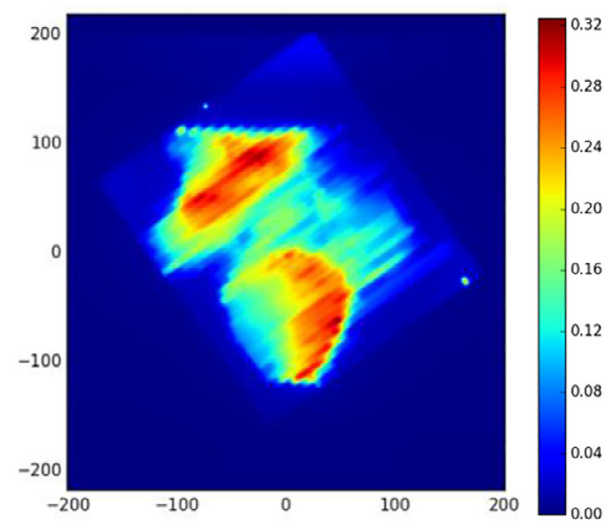

(b)

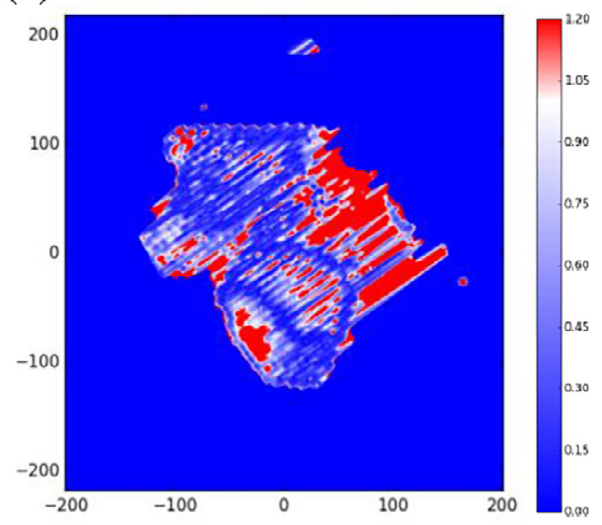

Pass rate $=75.1 \%$

(d)

Fig. 10. The (a) Measured, (b) Predicted, (c) global $3 \% / 3 \mathrm{~mm}$ gamma map and (d) local $2 \% / 2 \mathrm{~mm}$ gamma map for a VMAT field delivered on the BrainLab pelvis phantom. 
Table 11

The $3 \% / 3 \mathrm{~mm}$ gamma pass rate for the error plans (see Section 2.4.1) that were delivered on the BrainLab pelvis phantom.

\begin{tabular}{|c|c|c|c|c|c|c|}
\hline \multirow[t]{2}{*}{ Error } & & \multicolumn{5}{|c|}{$3 \% / 3 \mathrm{~mm}$ Gamma pass rate [\%] } \\
\hline & & POST & LPO & LAO & RAO & RPO \\
\hline No error & (original plan) & 99.9 & 98.2 & 100.0 & 99.9 & 98.1 \\
\hline \multirow[t]{5}{*}{ Dose } & MUs increased by $1 \%$ & 97.9 & 97.9 & 99.6 & 99.3 & 97.7 \\
\hline & MUs increased by $3 \%$ & 79.8 & 82.6 & 94.9 & 95.5 & 93.6 \\
\hline & MUs increased by $5 \%$ & 62.5 & 59.5 & 79.0 & 76.5 & 73.3 \\
\hline & MUs increased by $10 \%$ & 44.3 & 48.7 & 55.3 & 53.2 & 51.5 \\
\hline & MUs decreased by $5 \%$ & 97.9. & 94.7 & 83.1 & 71.3 & 84.0 \\
\hline \multirow[t]{2}{*}{ Gantry angle } & Offset by $+5^{\circ}$ & 97.8 & 99.0 & 94.1 & 91.3 & 98.6 \\
\hline & Offset by $+10^{\circ}$ & 98.1 & 94.7 & 79.6 & 68.8 & 97.8 \\
\hline \multirow[t]{8}{*}{ Set-up error } & Offset by $0.5 \mathrm{~cm}$ (right) & & & 100.0 & & \\
\hline & Offset by $1.0 \mathrm{~cm}$ (right) & & & 100.0 & & \\
\hline & Offset by $1.5 \mathrm{~cm}$ (right) & & & 99.7 & & \\
\hline & Offset by $2.0 \mathrm{~cm}$ (right) & & & 97.8 & & \\
\hline & Offset by $0.5 \mathrm{~cm}$ (ant) & & & 100.0 & & \\
\hline & Offset by $1.0 \mathrm{~cm}$ (ant) & & & 100.0 & & \\
\hline & Offset by $1.5 \mathrm{~cm}$ (ant) & & & 99.9 & & \\
\hline & Offset by $2.0 \mathrm{~cm}$ (ant) & & & 99.6 & & \\
\hline \multirow[t]{6}{*}{ MLC } & E1, Table 5 (not aligned) & 61.3 & 51.4 & 46.4 & 62.8 & 59.0 \\
\hline & E2, Table 5 & 79.5 & & & & \\
\hline & E3, Table 5 & & 47.9 & & & \\
\hline & E4, Table 5 & & & 98.3 & & \\
\hline & E5, Table 5 & & & & 14.5 & \\
\hline & E6, Table 5 & & & & & 94.3 \\
\hline
\end{tabular}

\section{Table 12}

The gamma pass rate and mean gamma at $3 \% / 3 \mathrm{~mm}$, mean dose difference and the dose difference at the centre of the EPID between the measured and predicted images for errors plans delivered on a $19 \mathrm{~cm}$ SW phantom.

\begin{tabular}{|c|c|c|c|c|c|}
\hline \multirow[t]{2}{*}{ Error } & & \multicolumn{2}{|c|}{$\begin{array}{l}3 \% / 3 \mathrm{~mm} \text { Gamma } \\
\text { test }\end{array}$} & \multirow{2}{*}{$\begin{array}{l}\text { Mean dose } \\
\text { difference } \\
\text {-[\%] }\end{array}$} & \multirow{2}{*}{$\begin{array}{l}\text { Dose } \\
\text { difference at } \\
\text { the centre of } \\
\text { EPID [\%] }\end{array}$} \\
\hline & & $\begin{array}{l}\text { Pass } \\
\text { rate } \\
{[\%]}\end{array}$ & $\begin{array}{l}\text { Mean } \\
\text { Gamma }\end{array}$ & & \\
\hline No Error & $\begin{array}{l}\text { (Original } \\
\text { plan) }\end{array}$ & 100 & 0.12 & 0.3 & 0.7 \\
\hline \multirow[t]{4}{*}{ Phantom size } & $\begin{array}{l}\text { Increased } \\
\text { by } 0.5 \mathrm{~cm}\end{array}$ & 100.0 & 0.26 & -1.7 & -1.6 \\
\hline & $\begin{array}{l}\text { Increased } \\
\text { by } 1.0 \mathrm{~cm}\end{array}$ & 95.1 & 0.5 & -2.9 & -3.7 \\
\hline & $\begin{array}{l}\text { Increased } \\
\text { by } 2.0 \mathrm{~cm}\end{array}$ & 48.2 & 1.03 & -5.5 & -8.1 \\
\hline & $\begin{array}{l}\text { Increased } \\
\text { by } 3.0 \mathrm{~cm}\end{array}$ & 33.8 & 1.62 & -7.8 & -12.1 \\
\hline
\end{tabular}

EPID model (aS1000) that they used in their study. In addition, Berry et al. used a field size specific backscatter correction that was previously developed for portal dosimetry by the same group [31]. In this work, no backscatter correction was used with the aS1200 since this EPID model has backscatter shield to remove the effect of uneven backscatter from the support arm [24].

It can be found from Fig. 6a and b that the effect of air gap is not negligible and the EPID signal can vary by up to $30 \%$ for the smallest air gap and largest field size and SW thickness. The $G_{c}$ factor was found to increase as the field size and thickness increases and as the air gap decreases. This could be due to the increase in secondary (scattered) photons that reach the EPID. We used a multi-dimensional look-up table to calculate the $G_{c}$ factor for a given field size, attenuator thickness and air gap as no fitting equation was found to model this factor accurately. The difference between the measured and calculated values for several images with a range of field sizes, SW thicknesses and air gaps was found to be less than $1 \%$ as seen in Table 8 .

As seen in Fig. $7 \mathrm{a}-\mathrm{c}$, the $G_{o}$ factor is relatively independent of field size and thickness, and is only affected by distance between the patient exit and EPID. Eq. (5) was found to accurately model this factor with $\mathrm{R}^{2}$ equal to 0.9994 as can be seen in Fig. 7c. The difference between the measured and modelled factor was found to be less than $1 \%$ at any point as seen in Fig. $7 \mathrm{~d}$.

Even though the effect of air gap on EPID central axis dose was studied by Talamonti et al. (2006) for pre-treatment IMRT verification [32], to the authors' knowledge, no one has introduced a model to correct the EPID in-axis and off-axis pixel response due to the change in the air gap between the patient and the imager.

The introduced model verified successfully for several IMRT and RA plans on up to three different phantoms. Most IMRT and RA fields passed the $3 \% / 3 \mathrm{~mm}$ gamma criteria by more than $99 \%$ as can be seen in Figs. 8 and 9 which is similar or superior to results published in previous studies $[14,15,33]$.

Berry et al. (2014) evaluated their model on 11 patients. They found that the average $5 \% / 3 \mathrm{~mm}$ gamma pass rate was increased from $89.1 \%$ to $95.7 \%$ by excluding all beams that interfere with the couch [23]. Since the couch structure was included in our model, no difference was noticed in gamma pass rate between beams that interfere and do not interfere with the couch. Therefore, the evaluation of the portal dose images in our model should not be influenced by beams transmission through the couch.

In order to determine the specificity and sensitivity of the system we both introduced deliberate errors and varied the gamma criteria. The high failure rate with a $2 \% / 2 \mathrm{~mm}$ gamma criteria (see Sections 3.4 .1 and 3.4.2), when no deliberate errors were presented, means that the specificity at this gamma criteria is too low for the system to be clinically useful. At $3 \% / 3 \mathrm{~mm}$, the specificity is sufficient for the test to be useable. The sensitivity at this level is discussed below. The low specificity of the system at $2 \% 2 \mathrm{~mm}$ gamma may be due to our not accounting for higher-order scattering components [34]. In addition, the effect of penumbra and inter-leaf leakage is more pronounced in the highly modulated treatments such as VMAT treatments [35]. It may be possible to improve the accuracy of the system by taking further measurements in order to produce a scatter kernel that, when convolved with the predicted image, will improve agreement with measurements. However, the $3 \% 3 \mathrm{~mm}$ gamma criteria used compares favourably to other published work. For example, Bedford et al. (2014) reported an average $3 \% / 3 \mathrm{~mm}$ global gamma pass rate from 9 VMAT plans equals to $93.7 \pm 3.0$ [14]. In addition, the $3 \% / 3 \mathrm{~mm}$ gamma pass rate in our method is considerably more than the action level of $90 \%$ per treatment field that was recommended in AAPM TG-119 for pre-treatment verification [36]. The differing patterns of failure for different beams shown in Table 11, discussed in more detail below, demonstrates that the failures are not due to systematic high or low predictions.

The predicted portal dose images for IMRT fields through different heterogeneous phantoms can be calculated accurately by our method, achieving $3 \% / 3 \mathrm{~mm}$ gamma pass rate of more than $99.5 \%$ on all the phantoms tested, as shown in Fig. 3. The geometries were simple, but featured large discontinuities and materials with different electron densities. Diagram b in Fig. 3 indicates that the presented method could be used for treatments where part of the field extends beyond the patient (e.g. breast treatments).

From the results reported in Table 11, the introduced method should be sensitive to dosimetric errors, the level of which is dependent on the gamma criteria used. The higher the dose criteria, the less sensitive the method will be to dose errors, as expected. Increasing the MUs by more than $3 \%$ caused all fields to have passing rates at $3 \% / 3 \mathrm{~mm}$ gamma criteria of less than $95 \%$. These results agreed with the results reported by Bedford et al. (2014) as all plans in their study fail the gamma evaluation when they increase the number of MUs by $10 \%$ [14].

The results in Table 11 demonstrate that in principle, our method should be sensitive to gantry angle errors, as the passing rates for some of the fields delivered in the prostate plans, for both $5^{\circ}$ and $10^{\circ}$ shifts, change markedly. For smaller angular rotations it is less likely to cause 
a noticeable drop in passing rates, as the smaller the rotation from the planned position the difference in the geometry that the beam transits. The precise level of gantry error that will be detected will depend on several factors including the specific patient anatomy and the location of fields with respect to the anatomy. This shows that our method should provide a way of detecting such an error, if a significant one were to occur.

The reported results from Table 11 show that, as might be expected, set-up errors of up to $2 \mathrm{~cm}$ shifts of the patient in one direction do not result in passing rates that would indicate that an error has occurred. This result agrees with the results reported by Bedford et al. [14]. While these set-up errors would result in significant errors for the patient in terms of dose localisation, the differences in the measured portal dose images are small because the anatomy that the beam passes through, for a prostate field shifted by $2 \mathrm{~cm}$ relative to the patient, does not change drastically. The sensitivity of the system to geometrical shifts will depend on the anatomy of the patient, for example, the presence of significant inhomogeneities in and around the treatment field, where greater inhomogeneity increases the likelihood of detecting positional errors. Our model was not intended to detect such set-up errors however, therefore the current protocols for treatment that are in place to produce the correct set-up of the patient, such as $\mathrm{kV}$ imaging, should be maintained in order to ensure this.

The results of the verification measurements performed on plans containing deliberate MLC errors, described in Table 11, demonstrate that the current method is sensitive to a range of MLC errors. This is consistent with the results reported by Bedford et al. [14]. The varied MLC errors introduced to the individual fields (E2 to E6 in Table 5) all produced distinct features in the gamma maps, making our model sensitive to these errors. However, the MLC errors introduced are unlikely to occur during treatment and more subtle MLC errors may not be detected by the presented model at all.

Table 12 reports the results of the field verification measurements performed with additional slabs of solid water added to the solid water slab phantom to simulate changes to the patient outline: they demonstrate that a change in water equivalent path length of $1 \mathrm{~cm}$ for an original path length of roughly $20 \mathrm{~cm}$ should be detectable for IVED. This type of error was not included in the study by Bedford et al. [14].

The precise thickness change that will result in a significant drop in the gamma passing rate will depend on the gamma criteria used, and the original path length of the beam through the patient; the larger the patient, the less changes in equivalent thickness will affect the measured dose at the EPID. Although the gamma pass rate did not drop enough to indicate an error for an additional $1 \mathrm{~cm}$ of SW, the error could be indicated via changes in the mean gamma, mean dose difference within the radiation field and the dose difference at the centre of the EPID between $m I s$ and $p I s_{p}$. Whilst patient weight changes can be detected during CBCT scans, our system has the benefit of being applicable to patients treated on a linac with an EPID, can be used for every fraction and does not result in an additional dose to the patient.

Although in-vivo EPID dosimetry has the potential to detect incidents which occur during treatments, many types of errors cannot be detected such as incorrect prescription and contouring. Therefore, it should be combined with other types of rules-based verification [37].

Using a fixed SID instead of a fixed air gap reduces the total treatment time since the EPID does not need to be moved for each beam to maintain the same air gap. In addition, it reduces the generation time of $p I s_{\text {air }}$ on Eclipse as only one verification plan is required to generate the pIs $s_{\text {air }}$.

The presented method is very simple to implement and can flag a number of significant errors for further assessment (one of the recommendations in "Toward Safer Radiotherapy" [5]). It has the potential to replace the pre-treatment verification for treatment plans with fields or arcs that can be fit on the portal imager and do not have a large couch rotation that restricts the use of the imager.

\section{Conclusion}

A model was introduced to perform in-vivo EPID dosimetry for IMRT, at fixed SID, and VMAT plans by adapting the methodology of Berry et al. (2012). A new correction factor was introduced to account for the change in air gap between the patient exit and EPID at each radiation field. To improve the sensitivity of the system, the couch model was included in the calculation of the equivalent thickness map, so the couch effect does not influence the gamma results. The introduced method was verified successfully on several IMRT and VMAT plans. The majority of fields/arcs passed the $3 \% / 3 \mathrm{~mm}$ gamma criteria by more than $95 \%$. Furthermore, relative to the methodology of Berry et al. (2012), our approach reduces the total treatment time and the time to generate portal dose images through air in Eclipse. These factors highlight the potential benefit of such a system as part of the radiotherapy pathway. Work is now in progress to evaluate the introduced model in the clinic.

\section{Appendix A. Supplementary data}

Supplementary data associated with this article can be found, in the online version, at https://doi.org/10.1016/j.ejmp.2018.07.010.

\section{References}

[1] Low DA, Moran JM, Dempsey JF, Dong L, Oldham M. Dosimetry tools and techniques for IMRT. Med Phys 2011;38:1313-38.

[2] Gupta T, Agarwal J, Jain S, Phurailatpam R, Kannan S, Ghosh-Laskar S, et al. Threedimensional conformal radiotherapy (3D-CRT) versus intensity modulated radiation therapy (IMRT) in squamous cell carcinoma of the head and neck: a randomized controlled trial. Radiother Oncol 2012;104:343-8.

[3] Wolff D, Stieler F, Welzel G, Lorenz F, Abo-Madyan Y, Mai S, et al. Volumetric modulated arc therapy (VMAT) vs. serial tomotherapy, step-and-shoot IMRT and 3D-conformal RT for treatment of prostate cancer. Radiother Oncol 2009;93:226-33.

[4] International Commission on Radiation Units and Measurements. ICRU report 83: prescribing, recording, and reporting photon beam intensity-modulated radiation therapy (IMRT). J ICRU 2010;10(1).

[5] Donaldson S. Towards safer radiotherapy. London: British Institute of Radiology. Institute of Physics and Engineering in Medicine, National Patient Safety Agency, Society and College of Radiographers, The Royal College of Radiologists; 2007.

[6] Mans A, Wendling M, McDermott L, Sonke J-J, Tielenburg R, Vijlbrief R, et al. Catching errors with in vivo EPID dosimetry. Med Phys 2010;37:2638-44.

[7] Mijnheer BJ, González P, Olaciregui-Ruiz I, Rozendaal RA, van Herk M, Mans A. Overview of 3-year experience with large-scale electronic portal imaging device-based 3-dimensional transit dosimetry. Pract Radiat Oncol 2015;5:e679-87.

[8] Huyskens D, Bogaerts R, Verstraete J, Lööf M, Nyström H akan, Fiorino C, et al. Practical guidelines for the implementation of in vivo dosimetry with diodes in external radiotherapy with photon beams (entrance dose) 2001

[9] Yorke E, Alecu R, Ding L, Fontenla D, Kalend A, Kaurin D, et al. Diode in vivo dosimetry for patients receiving external beam radiation therapy. Report of Task Group 2005;62

[10] Essers M, Mijnheer B. In vivo dosimetry during external photon beam radiotherapy. Int J Radiat Oncol Biol Phys 1999;43:245-59.

[11] Van Elmpt W, McDermott L, Nijsten S, Wendling M, Lambin P, Mijnheer B. A literature review of electronic portal imaging for radiotherapy dosimetry. Radiother Oncol 2008;88:289-309.

[12] Delaby N, Bouvier J, Jouyaux F, Barateau A, Lafond C. Validation of a transit EPID device for a clinical use: application to iViewDose (Elekta). Phys Med 2017;44:19-20.

[13] Stevens S, Dvorak P, Spevacek V, Pilarova K, Bray-Parry M, Gesner J, et al. An assessment of a 3D EPID-based dosimetry system using conventional two-and threedimensional detectors for VMAT. Phys Med 2018;45:25-34.

[14] Bedford JL, Hanson IM, Hansen VN. Portal dosimetry for VMAT using integrated images obtained during treatment. Med Phys 2014;41.

[15] Berry SL, Sheu R-D, Polvorosa CS, Wuu C-S. Implementation of EPID transit dosimetry based on a through-air dosimetry algorithm. Med Phys 2012;39:87-98.

[16] Piermattei A, Fidanzio A, Stimato G, Azario L, Grimaldi L, D’Onofrio G, et al. In vivo dosimetry by an aSi-based EPID. Med Phys 2006;33:4414-22.

[17] Wendling M, Louwe RJ, McDermott LN, Sonke J-J, van Herk M, Mijnheer BJ. Accurate two-dimensional IMRT verification using a back-projection EPID dosimetry method. Med Phys 2006;33:259-73.

[18] Wendling M, McDermott LN, Mans A, Sonke J-J, van Herk M, Mijnheer BJ. A simple backprojection algorithm for 3D in vivo EPID dosimetry of IMRT treatments. Med Phys 2009;36:3310-21.

[19] Olaciregui-Ruiz I, Rozendaal R, van Oers RF, Mijnheer B, Mans A. Virtual patient 3D dose reconstruction using in air EPID measurements and a back-projection algorithm for IMRT and VMAT treatments. Phys Med 2017;37:49-57. 
[20] Bedford JL, Hanson IM, Hansen VN. Comparison of forward-and back-projection in vivo EPID dosimetry for VMAT treatment of the prostate. Phys Med Biol 2018;63:025008.

[21] Van Elmpt W, Nijsten S, Mijnheer B, Minken A. Experimental verification of a portal dose prediction model. Med Phys 2005;32:2805-18.

[22] Van Esch A, Depuydt T, Huyskens DP. The use of an aSi-based EPID for routine absolute dosimetric pre-treatment verification of dynamic IMRT fields. Radiother Oncol 2004;71:223-34.

[23] Berry SL, Polvorosa C, Cheng S, Deutsch I, Chao KC, Wuu CS. Initial clinical experience performing patient treatment verification with an electronic portal imaging device transit dosimeter. Int J Radiat Oncol Biol Phys 2014;88:204-9.

[24] Miri N, Keller P, Zwan BJ, Greer P. EPID-based dosimetry to verify IMRT planar dose distribution for the aS1200 EPID and FFF beams. J Appl Clin Med Phys 2016;17:292-304.

[25] Moore AR, Warrington AJ, Aird EG, Bidmead AM, Dearnaley DP. A versatile phantom for quality assurance in the UK Medical Research Council (MRC) RT01 trial (ISRCTN47772397) in conformal radiotherapy for prostate cancer. Radiother Oncol 2006;80:82-5.

[26] Siddon RL. Fast calculation of the exact radiological path for a three-dimensional CT array. Med Phys 1985;12:252-5.

[27] Dearnaley D, Syndikus I, Mossop H, Khoo V, Birtle A, Bloomfield D, et al. Conventional versus hypofractionated high-dose intensity-modulated radiotherapy for prostate cancer: 5-year outcomes of the randomised, non-inferiority, phase 3 CHHiP trial. Lancet Oncol 2016;17:1047-60.

[28] The Institute of Cancer Research. International Randomized Study of Laparoscopic Prostatectomy vs Robotic Radiosurgery and Conventionally Fractionated Radiotherapy vs Radiosurgery for Early Stage Organ-Confined Prostate Cancer
(Clinical trial Protocol Number: ACCP003.2, version 4), https://www.icr.ac.uk/ourresearch/our-research-centres/clinical-trials-and-statistics-unit/clinical-trials/pace; 2013 [Accessed: 27.10.2017]

[29] Nelms BE, Chan MF, Jarry G, Lemire M, Lowden J, Hampton C, et al. Evaluating IMRT and VMAT dose accuracy: practical examples of failure to detect systematic errors when applying a commonly used metric and action levels. Med Phys 2013;40.

[30] White D, Booz J, Griffith R, Spokas J, Wilson I. ICRU Reports 44. J Int Commission Radiat Units Meas 1989.

[31] Berry SL, Polvorosa CS, Wuu C-S. A field size specific backscatter correction algorithm for accurate EPID dosimetry. Med Phys 2010;37:2425-34.

[32] Talamonti C, Casati M, Bucciolini M. Pretreatment verification of IMRT absolute dose distributions using a commercial a-Si EPID. Med Phys 2006;33:4367-78.

[33] Cilla S, Meluccio D, Fidanzio A, Azario L, Ianiro A, Macchia G, et al. Initial clinical experience with Epid-based in-vivo dosimetry for VMAT treatments of head-andneck tumors. Phys Med 2016;32:52-8.

[34] Spies L, Evans P, Partridge M, Hansen V, Bortfeld T. Direct measurement and analytical modeling of scatter in portal imaging. Med Phys 2000;27:462-71.

[35] Baeza JA, Wolfs CJ, Nijsten SM, Verhaegen F. Validation and uncertainty analysis of a pre-treatment 2D dose prediction model. Phys Med Biol 2018;63:035033.

[36] Ezzell GA, Burmeister JW, Dogan N, LoSasso TJ, Mechalakos JG, Mihailidis D, et al IMRT commissioning: multiple institution planning and dosimetry comparisons, a report from AAPM Task Group 119. Med Phys 2009;36:5359-73.

[37] Bojechko C, Phillps M, Kalet A, Ford EC. A quantification of the effectiveness of EPID dosimetry and software-based plan verification systems in detecting incidents in radiotherapy. Med Phys 2015;42:5363-9. 